\title{
Study on Dynamic Characteristics of a Secondary Regulated Loading System
}

\author{
Dengcheng Ma \\ Key Laboratory for Highway Construction Technology and Equipment of Ministry of Education \\ Chang'an University, Xi'an 710064, Shaanxi, China
}

Weiguo $\mathrm{Wu}$

Military Traffic and Transport Institute, 300161 Tianjin, China

Qiushuang Huang

Academy of Military Transportation, 300161 Tianjin, China

Shimin Yang \& Yongbiao Hu

Key Laboratory for Highway Construction Technology and Equipment of Ministry of Education Chang'an University, Xi'an 710064, Shaanxi, China

\begin{abstract}
Loading system is the core system of multi-functional engineering machinery as load vehicle to test traction performance of construction vehicle. For secondary regulation technology in loading system of multifunctional test platform, the mathematical models of electro-hydraulic servo valve, variable fuel tanks, secondary component were established by modeling method for the secondarily regulated loading system. By using the PID controller to adjust the parameters, mainly from step loading model and sine wave loading model to analysis the simulation. Studying on dynamic characteristics and reliability of the secondary regulation technology system for loading control system of test-platform. The results show that the construction machinery multi-function test-platform uses the secondary regulation technology system is feasible, the secondary regulation technology can meet the performance requirements of the loading system.
\end{abstract}

Keywords: Secondary regulation, Test Platform, Dynamic characteristics, Simulation

\section{Introduction}

In order to study the construction vehicle traction performance, in the laboratory soil tests, usually using simulated loading trucks on the tractor, to reproduce the load during towing operations. The multi-functional engineering machinery test platform can be used as load car for traction vehicle, the test platform is designed by Chang'an University in China, shown in Fig.1. The load imposed by the test platform is controlled through hydrostatic secondary regulated loading system (shown in Figure 2). Traction load imposed by vehicles controlled by secondary regulated loading system, the running wheels of load car are driven by the secondary component, when the system loads, the Secondary component impose a load torque shaft to the test platform. In loading system, constant pressure variable pump and electrical connections, the load absorbed power is converted to electrical energy back to the power grid. In order to study the construction vehicle traction performance, Applied traction load, including the step load, sinusoidal load and measured load spectrum, etc. The literature studies have shown that construction vehicle traction load is often volatile, and its dominant frequency is generally less than $6 \mathrm{~Hz}$ (Yao, Huai-xin, 2002), so secondary regulated loading system should meet this requirement, and has a degree of stability nature, it is necessary to study the dynamic characteristics of hydrostatic secondary regulated loading system.

\section{Secondary regulated loading system}

Hydrostatic secondary regulation technology is different from the first element regulation technology. The latter is the regulation of hydraulic pump, but the former is directly regulated variable motor (Zhan, Xing-qun., Zhang, Yan-hua., \& Zhao, Ke-ding, 2000)(Peng, Song., Ding, Qiang., \& Qiu, Li-hua. 2006)(Zhan, Xingqun., Zhang, Yanhua., \& Zhao, Keding. 2000). The technology used to load truck loading system, load truck axle corner 
(position), speed, torque and power can be directly adjusted. When the system is loading, the characteristics of the torque characteristics of the secondary component unit meet the load requirement.

Shown in Fig.2, the system uses Hydrostatic secondary regulation technology, the basic principle is: constant network is formed through constant pressure controller and the constant pressure accumulator. The second constant voltage regulation circuit is composed of the constant pressure variable hydraulic pump and the variable motors (optional four-quadrant operation can be of secondary components), in the loop with accumulator group, in order to maintain constant pressure, and the constant pressure variable pump is driven by DC motor. The secondary component (the variable motor) is controlled through the secondary component controller. The oil of the constant pressure variable pump is supplied by the gear pump, while fill oil in low side. When the multi-function test bed is used of load vehicle, the secondary component is used as hydraulic pumps, and the power recovery network.

\section{The mathematical model of loading system}

In order to study the dynamic characteristics of loading system, the various components of the dynamic loading system mathematical model are need to be established and the combination of mathematical models of various components is the system of mathematical models. Hydrostatic secondary regulated loading system loading system is composed of the secondary component unit and constant oil source, and constant oil source is composed of hydraulic pump and the accumulator. Secondary component unit include electro-hydraulic servo valve, variable control cylinder, the second component, and the second component controller. Variable control fuel tank is form a small closed-loop position through position sensor(Zhan, Xingqun., Zhang, Yanhua., \& Zhao, Keding. 2001).

\subsection{Electro-hydraulic servo valve}

When $I$ is the electric current, as an input parameter. $Q_{s v}$ is the out flow of electro-hydraulic servo valve, as an output parameter. $\omega_{s v}$ is the natural frequency of electro-hydraulic servo valve, $\zeta_{s v}$ is the damping ratio of electro-hydraulic servo valve, $K_{s v}$ is the flow gain of electro-hydraulic servo valve, $G_{s v}$ is the second-order transfer function approximation of Electro hydraulic servo valve. Because most of the electro-hydraulic servo systems, electro-hydraulic servo valve dynamic performance is often higher than the dynamic component (secondary component) of the dynamic response. Loading system bandwidth due to the lower, while the electro-hydraulic servo valve is greater than the bandwidth $100 \mathrm{~Hz}$, considering a certain overshoot, so the electro-hydraulic servo valve second-order transfer function simplified oscillation link. It can be expressed using Eq.(1), respectively(Wang, Chunxing. 1990).

$$
G_{s v}(s)=\frac{Q_{s v}(s)}{I(s)}=\frac{K_{s v}}{\frac{s^{2}}{\omega^{2}}+\frac{2 \zeta_{s v}}{\omega_{s v}} s+1}
$$

As the natural frequency of electro-hydraulic servo valve much higher than the secondary component, generally consider it to be the proportion of links, the expression for the Eq.(1) becomes:

$$
G_{s v}(s)=\frac{Q_{s v}(s)}{I(s)}=K_{s v}
$$

\subsection{Variable Control fuel tank}

When $Q_{L}$ is the flow of variable control fuel tank, as the input parameter. $X_{p}$ is the piston displacement of variable s control fuel tank, as the output parameter. $P_{L}$ is the two-cavity pressure of variable control fuel tank, as the middle of the two cavity parameters. They can be calculated using Eq.(3), respectively(Yu, Feng., Liu, Jiahui., Pu,Hong., \& Zhang, Jihai. 2000).

$$
Q_{L}=A s X_{p}+C_{t c} P_{L}+\frac{V_{t}}{4 \beta_{e}} s P_{L}
$$

where $A$ is the an effective role in an area of variable control fuel tank, $C_{t c}$ is the total leakage factor of variable control fuel tank, $V_{t}$ is the total volume of cavity of variable control fuel tank, $\beta_{e}$ is the work of oil in bulk modulus.

Eq.(4)is the force balance equation of variable control fuel tank: 


$$
A P_{L}=m \frac{d^{2} X_{P}}{d t^{2}}+B_{P} \frac{d X_{P}}{d t}+K X_{P}+F_{L}
$$

When excluding foreign interference load and friction, Laplace transformation, the expression for the force balance equation of variable control fuel tank becomes:

$$
A P_{L}=\left(m s^{2}+B_{P} s+K\right) X_{P}
$$

Where $m$ is the equivalent quality of variable control fuel tank piston, $B_{P}$ is the piston and load viscous damping coefficient of variable control fuel tank, $K$ is the spring stiffness of the load, $F_{L}$ is the outside interference load and friction on the piston.

Because the volume of variable control fuel tank is very small, it can not be counted by the volume change caused by compression of the flow changes. In addition, the fuel tanks are sealed and the servo pressure on the lower shell, so the total leakage factor of variable control fuel tank can not be counted. The frequency response of variable control fuel tank close to $100 \mathrm{~Hz}$, much higher than the second-conditioning systems, so variable control fuel tank can be simplified as integral part of the link. It can be expressed using Eq.(6).

$$
G_{y s}(s)=\frac{X_{p}(s)}{Q_{L}(s)}=\frac{1}{A s}
$$

\subsection{The secondary component}

When $X_{P}$ as an input parameter and $V$ as the output parameter for the secondary component, $\gamma$ is the Swash plate angle of the Secondary component, Generally around $10^{\circ}$. Within this context, $\tan \gamma=X_{P} / r_{a} \approx \gamma$, so $\tan \gamma$ can replaced by $\gamma . r_{a}$ is the effective radius of the swash plate for secondary component. $G_{\gamma x p}(s)$ is the transfer function for $X_{P}$ to $V$, it can be expressed using eq.(6).

$$
G_{\gamma x p}(s)=\frac{\gamma(s)}{X_{P}(s)}=1 / r_{a}=K_{\gamma x p}
$$

Where $K_{\gamma x p}$ is the Conversion factors for switch $X_{P}$ to $\gamma$. Eq.(7) is the equation of secondary component displacement: $V=\frac{V_{\max }}{\gamma_{\max }} \gamma \approx \frac{V_{\max }}{X_{p \max }} X_{p}$

Where $V_{\max }$ is the maximum displacement of the secondary component, $X_{p \max }$ is the maximum displacement of the variable control fuel tank, $\gamma_{\max }$ is the maximum swash plate angle of the secondary component.

When the secondary component loads, the working conditions in the pump, $M_{L}$ is the input torque of secondary component. It can be calculated using Eq.(8).

$$
M_{L}=\frac{V_{\max }}{X_{P \max }} X_{P} P_{s} \eta_{h}+J_{s}^{2} \theta+B s \theta
$$

Where $P_{s}$ is the pressure of Constant Pressure System, $\eta_{h}$ is the efficiency of the Secondary component, $\theta$ is the axis angle of the Secondary component, $J$ is the total moment of inertia of the secondary component in Pump operating conditions, and $B$ is the total viscous damping coefficient of the secondary component in Pump operating conditions.

\subsection{The secondary component controller}

When $U(s)$ is the input voltage of the controller, and the mathematical model of position sensor is $U(s)=K_{W} X_{P}(s)$

Where $K_{W}$ is the feedback coefficient of the position sensor.

The mathematical model of the controller is $I(s)=G_{C}(s) U(s)$ (10),respectively(Yu, Feng., Liu, Jiahui., Pu, Hong., \& Zhang, Jihai. 2000). 
Where $I(s)$ is the input current of electro-hydraulic servo valve, $G_{C}(s)$ is the transfer function of the controller, and the mathematical model of torque sensor is $U_{f}(s)=K_{F} M(s)$.

Combination of Eqs(1) to (9), resulting in a simplified closed-loop transfer function of secondary component unit block diagram, shown in Fig.3. In addition, constant pressure oil source is composed of Constant pressure variable pump and accumulator, the mathematical model of Constant pressure variable pump and the accumulator see reference (Zhan, Xingqun., Zhang, Yanhua., \& Zhao, Keding. 2001). As the constant pressure oil source has good dynamic characteristics, in order to simplify the analysis, we think that the constant voltage source of oil without pressure fluctuations, and pressure is constant value, so the mathematical model of constant pressure oil source is not discussed. In the following simulation, the simplified mathematical model is used for the secondary component unit load system mathematical model.

\section{Simulation analysis and results}

Combination of the above-mentioned mathematical model of the transfer function, the closed-loop transfer function of secondary component unit is simulated by Matlab/Simulink simulation software (Simulation shown in Figure 4), Key analytical step load and sinusoidal load (frequency adjustable) two kinds of loading mode. The simulation parameters of the components are determined by design information of each component for Load vehicle hydraulic system and other relevant documents, as shown in Table 1(Wei, Zhanfang. 2004).

\subsection{The dynamic response to step load}

For the system without additional correction, the Step load response time can not meet the requirements of the loading vehicle,so use the PID controller to adjust parameters to optimize the system. When $T_{d}, T_{i}$ and $K_{p}$ are PID controller parameters, Selects $K_{p}=3, T_{d}=0.01125, T_{i}=0.05$. Load step load (amplitude 100N * $\mathrm{m})$ by the simulation curve shown in Fig. 5.Fig.5 shows that system adjustment time is 0.06 second and the overshoot of amplitude is percent 17. They can meet the vehicle load on the loading accuracy. Fig. 6 is the closed-loop Bode amplitude-frequency diagram of system. Fig.6 shows that the bandwidth of the system is $305 \mathrm{rad} / \mathrm{s}(49 \mathrm{~Hz})$, and it is greater than the main frequency of load fluctuations for construction vehicle. After PID adjustment, the system has good dynamic characteristics and control performance, and it can meet the load car load requirements.

\section{2 sine load dynamic response}

When input $6 \mathrm{HZ}$ sine wave signal (amplitude $500 \mathrm{~N} * \mathrm{~m}$ ) of loading system, the response curve is obtained, shown in Fig.7.Fig. 7 shows that the system tracking a frequency of $6 \mathrm{~Hz}$ input signal precision is high, according to (Yao, Huai-xin. 2002) on the construction machinery traction load analysis, the working resistance frequency of construction vehicle range typically $1 \mathrm{~Hz} \sim 5 \mathrm{~Hz}$, so the secondary regulation loading system can be applied to construction machinery multifunctional test platform (loading trucks), which can meet the needs of the actual load.

\section{Conclusions}

Based on the theoretical analysis of the loading system, a simulation system about the loading system is built up and the simulation is also done. Through the simulation, some conclusions can be achieved as follows.

(1) Hydrostatic secondary regulation system is used to loading system of test platform, to avoid the traditional loading process, due to pressure changes caused by load changes, leading to load instability, experimental data is not reliable.

(2) The time for system adjustment is 0.06 second and the overshoot of amplitude is percent 17 , they can meet the test platform loading system on the loading accuracy.

(3) When input $6 \mathrm{HZ}$ sine wave signal of loading system, the tracking accuracy is high, and the loading system has good dynamic performance and control stability.

(4) Because the working resistance frequency of construction vehicle range typically $1 \mathrm{~Hz} \sim 5 \mathrm{~Hz}$, so the secondary regulation loading system can be applied to construction machinery multifunctional test platform (loading trucks), and meet the needs of the actual load.

Acknowledgement

This research has been financed by the Project of Natural Science Foundation of Shann'xi Province of China (2007E21). 


\section{References}

Peng, Song, Ding, Qiang, \& Qiu, Li-hua. (2006). Study on Control Characteristics of the Secondary Regulation Load Simulation Test Equipment for the Construction Equipment Hydraulic Chassis. Machine Tool and Hydraulic, 2006(11),110-115.

Wang, Chunxing. (1990). Hydraulic Servo Control System.Beijin: Machinery Industry Press, 1990.

Wei, Zhanfang. (2004). Study on Simulation Test Equipment for the Construction Equipment Hydraulic Chassis. Chang'an University master's thesis, 2004.

Yao, Huai-xin. (2002). Construction equipment chassis and the hydraulic transmission theory-- Running and control of hydraulic machinery. Beijin: Communications Press, 2002.

Yu, Feng, Liu, Jiahui, Pu, Hong, \& Zhang, Jihai. (2000). Study of Double Closed Loop Control Characteristics of Hydrostatic Transmission System with Secondary Regulation. Journal of Jiamusi University (Natural Science Edition),2000(6),103 105.

Zhan, Xing-qun., Zhang, Yan-hua., \& Zhao, Ke-ding. (2000). Decouple Control for Torque-Loading Equipment with Hydrostatic Driving Secondary Regulation Technology. Journal of shanghai jiao tong university, 2000,34(11),1501-1503.

Zhan, Xingqun, Zhang, Yanhua, \& Zhao, Keding. (2000). Hardware Decouple Methods for Torque-Loading Equipment with Hydrostatic Driving Secondary Regulation Technology. Journal of shanghai jiao tong university, 2000(11),1497 1500.

Zhan, Xingqun, Zhang, Yanhua, \& Zhao, Keding. (2001). Experiments on a secondary Regulated Loading System.engineering science, 2001,3(11),70 73.

Table 1. Simulation Parameters

\begin{tabular}{|c|c|c|c|c|c|}
\hline $\begin{array}{c}\text { Parameter } \\
\text { Name }\end{array}$ & Units & Value & $\begin{array}{c}\text { Parameter } \\
\text { Name }\end{array}$ & Units & Value \\
\hline$A$ & $m^{2}$ & $5.45 \times 10^{-4}$ & $\mathrm{~V}$ & $\mathrm{~m}^{3}$ & $2.2 \times 10^{-5}$ \\
\hline$K_{c}$ & $N / m$ & 5292 & $C_{t c}$ & $\mathrm{~m}^{3} / \mathrm{s} \cdot \mathrm{P}_{a}$ & $5 \times 10^{-13}$ \\
\hline$\beta_{e}$ & $N / \mathrm{m}^{2}$ & $7 \times 10^{8}$ & $m$ & $\mathrm{~kg}$ & 5 \\
\hline$\xi_{v}$ & & 0.91 & $\omega_{v}$ & $\mathrm{rad} / \mathrm{s}$ & 1178 \\
\hline
\end{tabular}

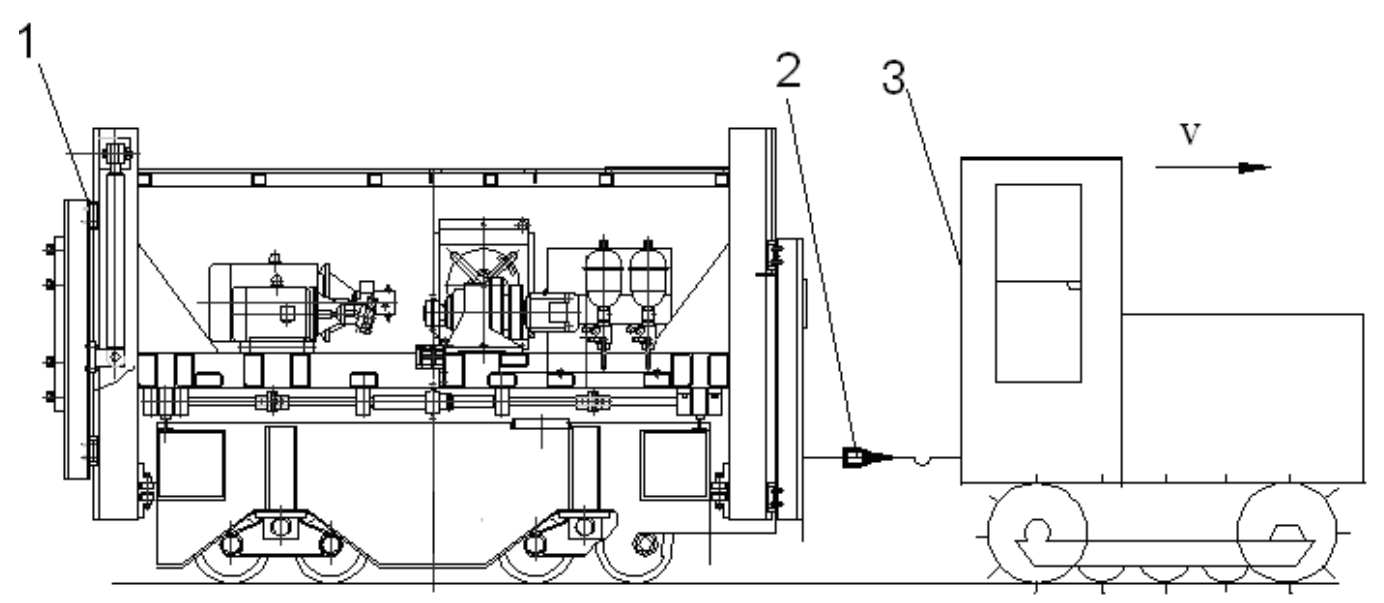

1. Load car; 2. Traction hook (with tension sensors); 3.Engineering tractor

Figure 1. Schematic diagram of load car load 


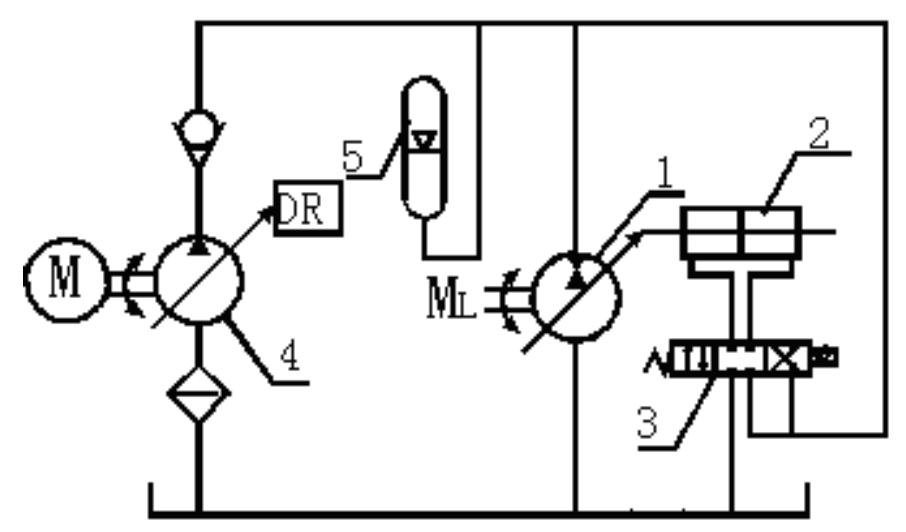

1. Secondary component; $\quad$ 2. Variable cylinder; $\quad 3$. Electro-hydraulic servo (proportional) valve; 4. Constant pressure variable hydraulic pump; 5. Hydraulic accumulator

Figure 2. Hydrostatic secondary regulation loading system Schematic

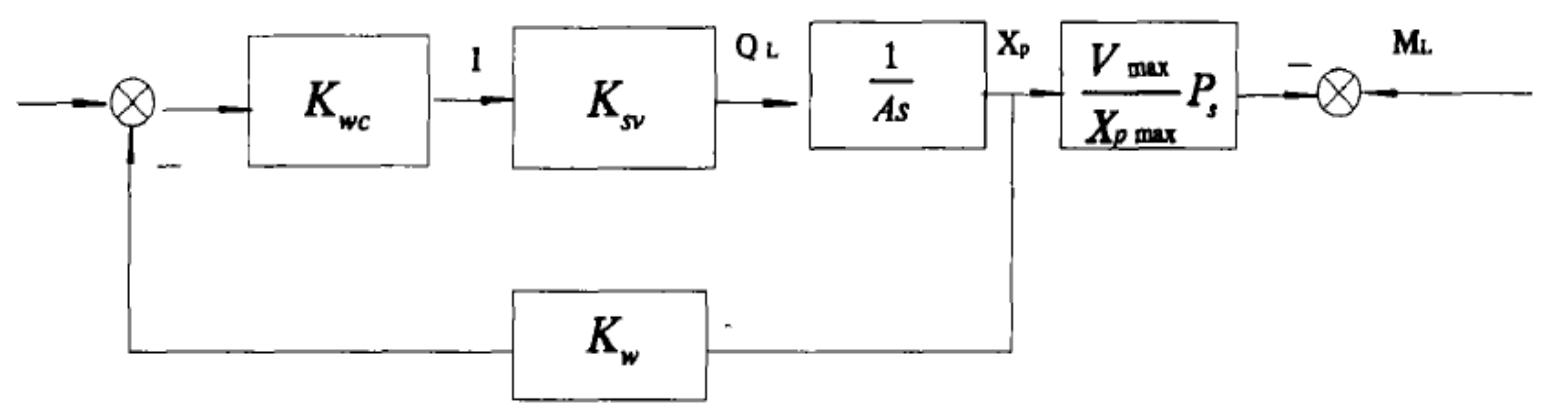

Figure 3. the simplified closed-loop transfer function of secondary component unit block diagram

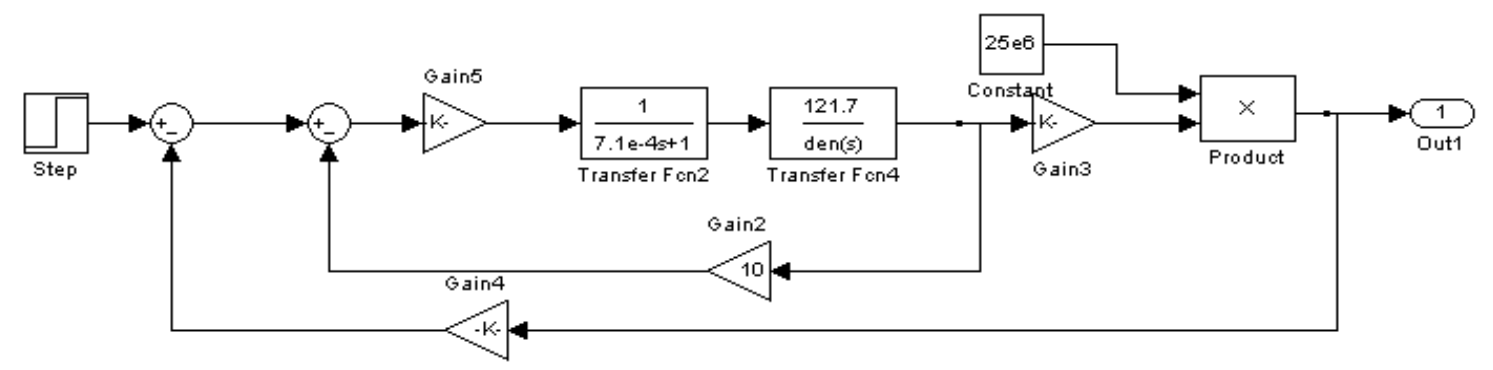

Figure 4. the simulation diagram of secondary component unit 


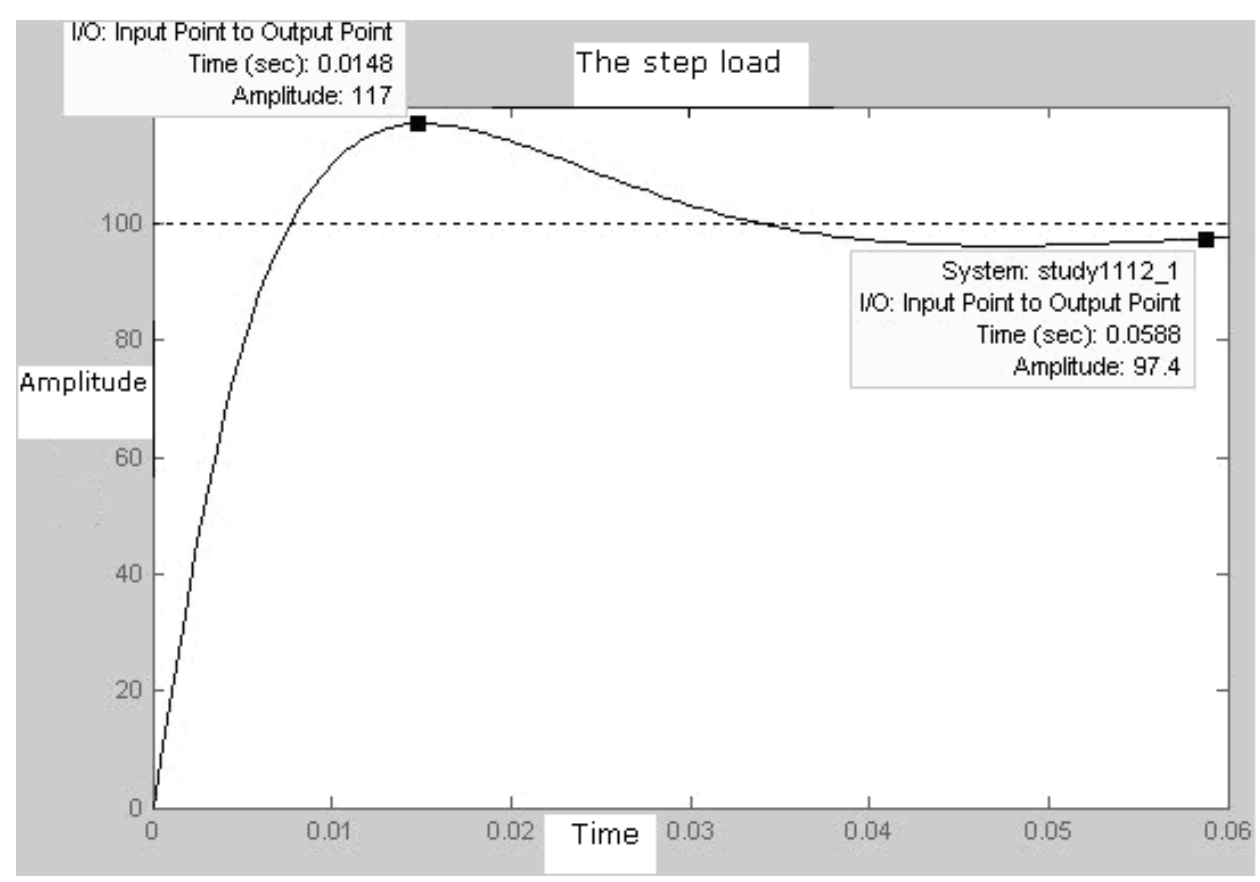

Figure 5. The step load response curve

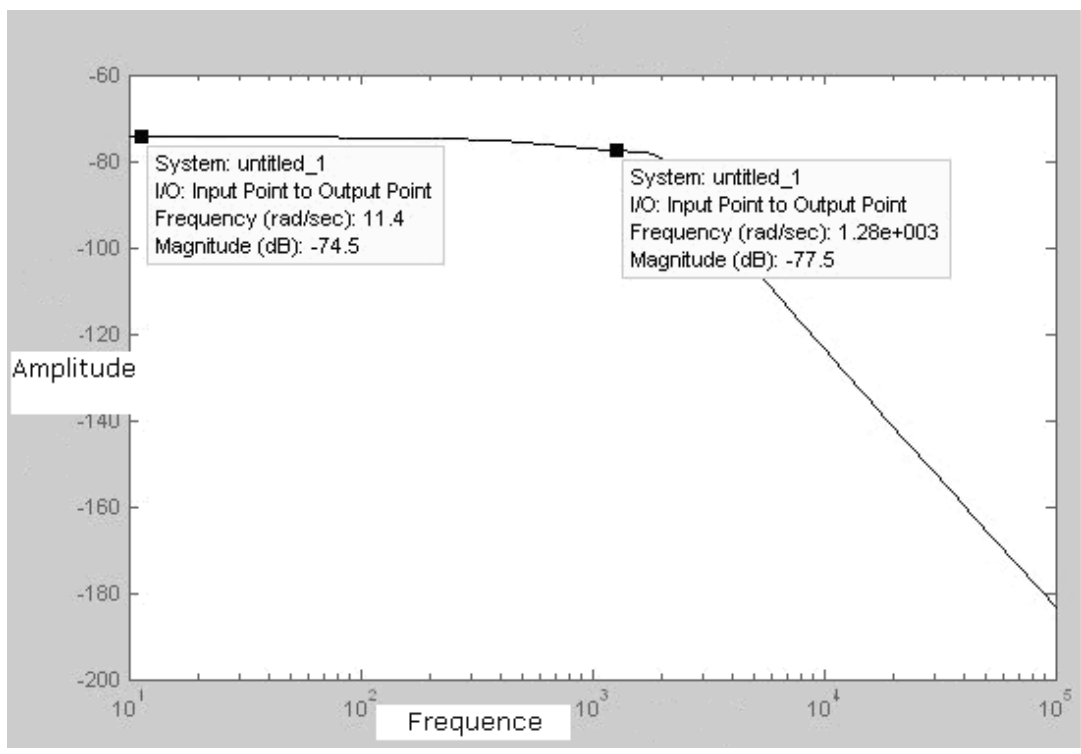

Figure 6. The closed-loop amplitude-frequency diagram 


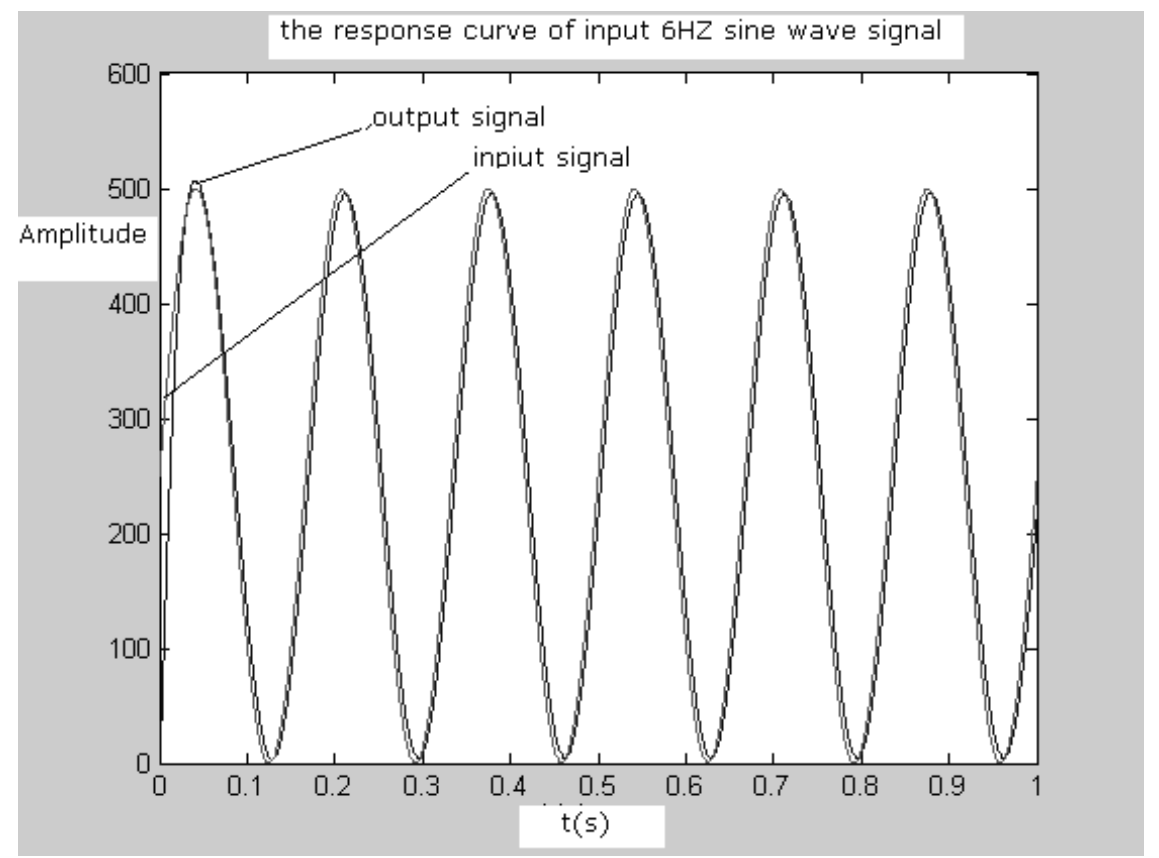

Figure 7. the response curve of input $6 \mathrm{HZ}$ sine wave signal 